\title{
Reactive Lesions of the Gingiva: Diagnosis and Treatment Options
}

\author{
Jeffrey A. Rossmann* \\ Department of Periodontics, Texas A \& M Health Science Center, Baylor College of Dentistry, Dallas, Texas, USA
}

\begin{abstract}
Reactive lesions found on the gingiva are common and tend to be non-neoplastic growths. They are usually not painful and are often overlooked by the patient until they become symptomatic or are identified by their dental healthcare provider. Since they may be present for weeks to months, it is common to see ulceration of the surface tissue due to trauma. They have a recurrence rate of $5-20 \%$ after excision depending on the diagnosis, the completeness of the surgical removal, and the ability to address the local irritating factors associated with their etiology. It is recommended to obtain histologic diagnoses for the lesions since their clinical appearances can be similar amongst various lesions, but the recurrence rates are different. The treatment for all common reactive lesions includes surgical excision by scalpel, laser, or radial/ electrosurgery. Treatment should also include removal of the underlying etiology through curettage of tooth accretions, replacement of defective restorations, and elimination of traumatic habits. Regular dental hygiene maintenance with professional follow-up care should reduce the incidence of recurrence for most types of gingival lesions. This review of identification and treatment of gingival lesions includes the newly recognized localized juvenile spongiotic gingival hyperplasia, which should become part of the differential diagnosis for reactive lesions in juvenile patients.
\end{abstract}

Keywords: Reactive lesions, carbon dioxide laser, mucogingival defects, surgical excision.

\section{INTRODUCTION}

Growths of the gingival tissues are common and often result from underlying systemic disease, drug-induced stimulus, local iatrogenic factors, and dental plaque. The lesions described in this review are considered reactive lesions that are non-neoplastic in nature and not implicated with drug involvement. The duration of the lesion is often weeks to months due to the slow growth and mild symptoms; they are rarely painful, but often interfere with adequate plaque control. Since their duration is long, it is not uncommon to see ulceration to the epithelial surface from trauma. This paper will emphasize the clinician's viewpoint in identification, diagnosis, and treatment for these common reactive lesions of the gingiva. The use of the carbon dioxide laser has become an accepted and sometimes preferred mode of therapy for gingival lesions due to the unique characteristics of the wound and ease of use of the laser. A review of the pertinent factors for a successful outcome in using the laser will be explored. Case reports will supplement the description of each lesion to allow a better understanding for the clinician in developing a differential diagnosis.

The terminology used in this review is by Neville [1] classifying the common gingival overgrowth lesions under the following categories: 1) Pyogenic granuloma (including pregnancy tumor); 2) Peripheral ossifying fibroma (also referred to as ossifying fibroid epulis, peripheral fibroma with calcification, calcifying fibroblastic granuloma, and peripheral odontogenic ossifying fibroma); 3) Peripheral fibroma also referred to as fibrous hyperplasia, fibrous

*Address correspondence to this author at the Department of Periodontics, TAMHSC - Baylor College of Dentistry, 3302 Gaston Avenue, Room 141, Dallas, Texas 75246, USA; Tel: 214828 8140; Fax: 214874 4532;

E-mail: jrossmann@bcd.tamhsc.edu epulis); and 4) Peripheral giant cell granuloma. Included in this review is a recently recognized entity which was first described as juvenile spongiotic gingivitis [2] and then as localized juvenile spongiotic gingival hyperplasia [3]. The lesions referred to above are considered reactive and arise in response to local irritants such as defective restorations, dental plaque, calculus, trauma, and iatrogenic factors. They are not considered neoplasms and have distinctive histopathology to identify their diagnosis. Although benign in nature they do have a tendency towards recurrence with incomplete removal of the lesion or the local irritants involved at the site. The treatment in each case is surgical excision; however, different treatment modalities may offer better outcomes with less frequency of recurrence. In addition, the complete excision of the lesion will often result in a cosmetic or functional defect leaving the site without keratinized gingiva and with root exposure. Therefore, the ability of the clinician to obtain a good outcome involves repair of the mucogingival defect as well as complete excision of the lesion. This can usually be accomplished in one surgical session with proper planning. The identification of the lesion must be confirmed histologically since they often show similar clinical appearance.

The prevalence of reactive lesions of the gingiva is reported to be rather common with the peripheral fibroma being the most common category $(56-61 \%)$ followed in descending order by the pyogenic granuloma $(19-27 \%)$, peripheral ossifying fibroma $(10-18 \%)$, and peripheral giant cell granuloma $(1.5-7 \%)$ based on over 3000 cases studied $[4,5]$. Localized juvenile spongiotic gingival hyperplasia is a newly defined lesion of young patients that has gone undiagnosed in the past or was simply considered a reactive gingivitis [3]. All of these lesions are more commonly seen in females with only the peripheral giant cell granuloma having no consistent sex predilection. 


\section{PYOGENIC GRANULOMA}

The pyogenic granuloma (PG) is an exuberant tissue response to local irritation or trauma; it is not a neoplasm. The young lesions are highly vascular, red or reddish purple, often elevated and ulcerated, and bleed easily. Older lesions tend to be more collagenized and pink in appearance. Some believe the older PG lesion will develop into a peripheral ossifying fibroma or peripheral fibroma over time through fibrous maturation and ossification [1,6,7]. The PG and pregnancy tumor have an identical histologic appearance and the pregnancy tumor is considered a PG occurring in pregnant females. However, Daley et al. reported on 42 lesions diagnosed as pregnancy tumors and felt the entity deserved its own distinction based not on histologic features, but on etiology, biologic behavior and treatment protocol [8]. The PG can often exhibit rapid growth, appearing on the gingiva in $75 \%$ of reported cases with a female predilection. The pregnancy tumor tends to increase in incidence with increasing hormonal levels of estrogen and progesterone up to the seventh month of gestation when the critical stimulatory concentration is reached.

The histological appearance is characterized by vast

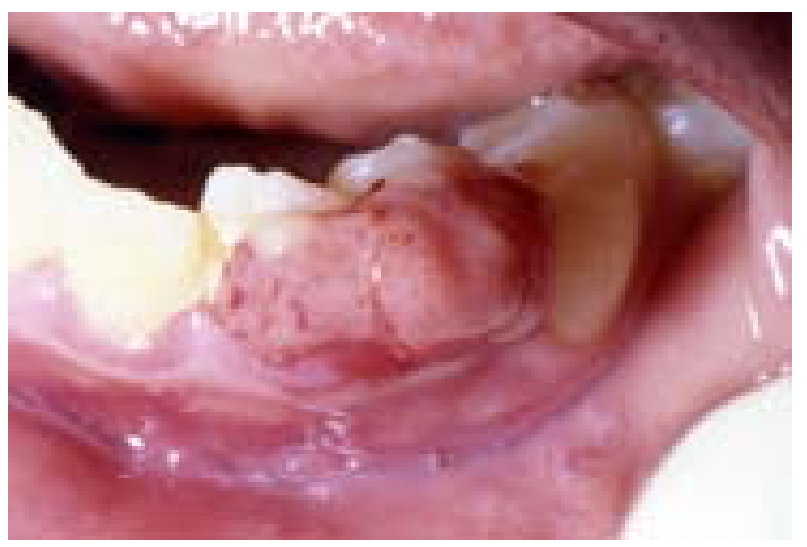

a

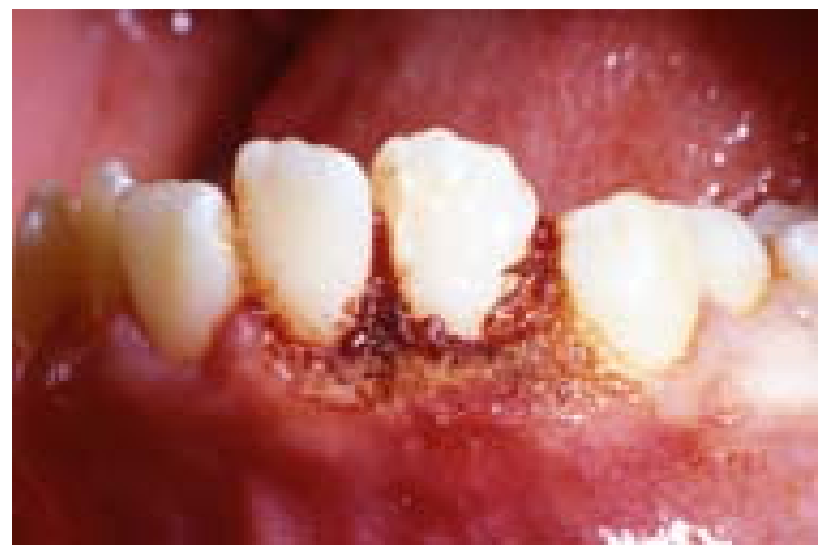

C numbers of endothelium lined vascular spaces infiltrated with lymphocytes, plasma cells and neutrophils. There is extensive fibroblastic proliferation with a diffuse, often dense chronic inflammatory infiltrate. The lesion is covered by a thin, often ulcerated layer of stratified squamous epithelium. Despite the name, no pyogenic material or pus is found in the lesion.

Treatment involves complete excision of the lesion down to the periosteum or periodontal ligament and removal of local irritants. This can be difficult due to the hemorrhagic nature of the lesion and may be better accomplished through laser eradication than the scalpel (Fig. 1). This 32 year old female presented in the second trimester of her pregnancy with a rapidly growing lesion on the facial gingiva of the lower anterior teeth causing an inability to achieve plaque control and eat properly. The lesion was removed under local anesthesia using the carbon dioxide laser and submitted for histologic analysis. Once adequate plaque control was achieved post-surgically the patient did not suffer any recurrence of the PG.

The carbon dioxide laser has been used effectively for surgical excision. The advantages include surgical visibility

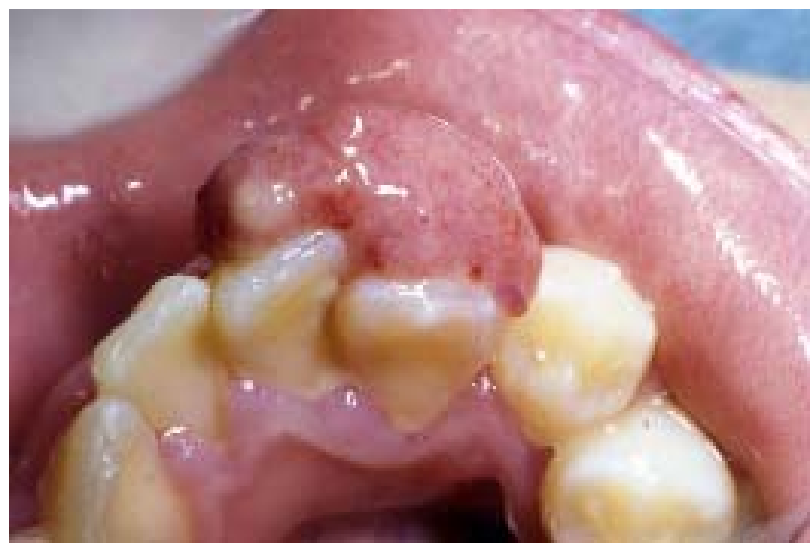

b

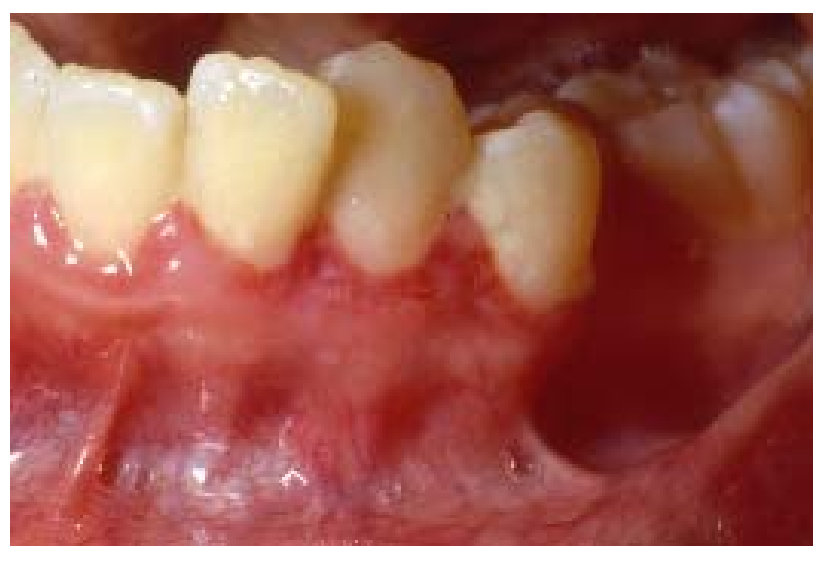

d

Fig. (1). Pyogenic granuloma. (a) Initial presentation of a pyogenic granuloma at the mandibular anterior teeth, facial view. (b) Occlusal view. (c) Surgical excision by $\mathrm{CO}_{2}$ laser (note dry field and excellent visibility). (d) Seven days post-surgery showing minimal inflammation. 
since minor vessels are sealed with the laser ablation (up to $0.5 \mathrm{~mm}$ diameter) and sterility of the field during surgery. The laser also leaves a layer of carbonization (char) over the wound to provide a biologic bandage that seals the surface for additional post-surgical comfort without the need for suture nor placement of a palliative dressing [9-11]. The carbon dioxide laser energy is also highly absorbed in water causing cellular disintegration through vaporization of the cellular fluids. This may reduce the recurrence rate through destruction of the cells implicated in the origin of the PG (Fig. 1). A recurrence rate between $16-21 \%$ has been reported for PG with conventional excision techniques $[5,12]$. The recurrence rate is increased if the lesion is removed during pregnancy due to the stimulatory effects of the female sex hormones, and it is advised to wait until postpartum if possible. The timing of treatment often depends upon whether the lesion is causing functional or esthetic concerns for the patient during pregnancy [8]. Anecdotal evidence suggests the recurrence rate is less with laser excision, however, clinical trials have not been reported to substantiate that claim.

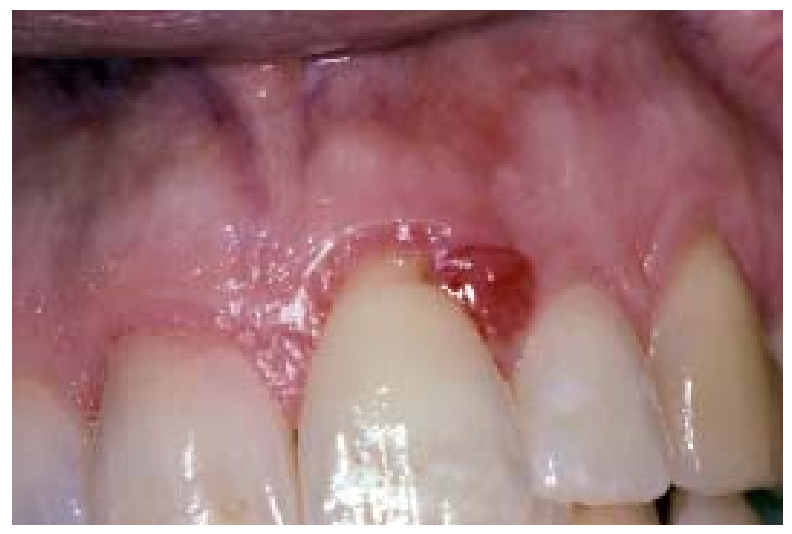

a

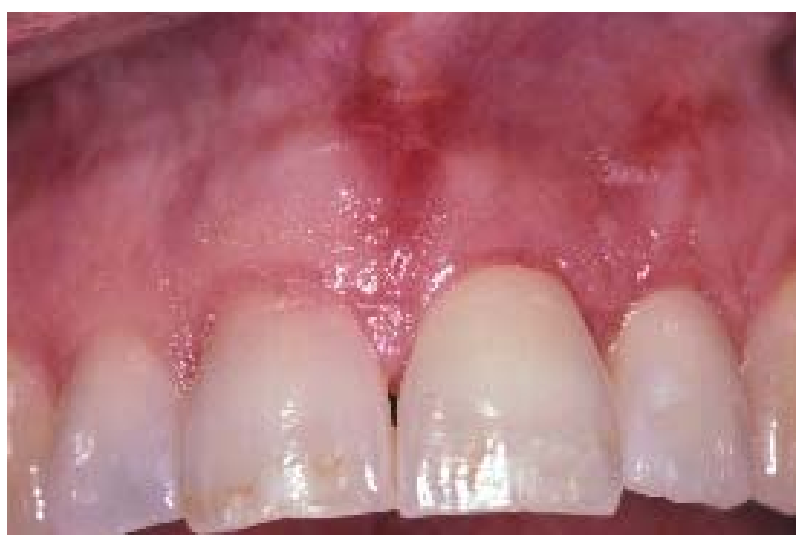

C
This 45 year old female presented with a three month history of a red lesion on the gingiva between teeth $\# 9$ and 10 (Fig. 2). Additionally, gingival recession of $2 \mathrm{~mm}$ was noted on the facial of tooth \#9. Surgical excision of the lesion was accomplished with a scalpel incision combined with coronal positioning of a full thickness mucoperiosteal flap to cover the recession defect. The healed site shows a good esthetic result and no recurrence of the lesion over the past nine years has occurred.

\section{PERIPHERAL FIBROMA}

The peripheral fibroma (PF) is the most common reactive lesion often resulting from trauma or local irritation $[5,13]$. It originates from stimulated connective tissue of gingival origin and has been referred to as a gingival epulis. The lesion appears as a raised mass that is pedunculated or sessile with a smooth surface, and is usually the same color as the surrounding gingiva. They are commonly found in the interdental papilla of the anterior teeth in adults (fourth to sixth decade of life) with a slight female predilection. Sixty percent of the time, the PF is primarily found in the maxilla.

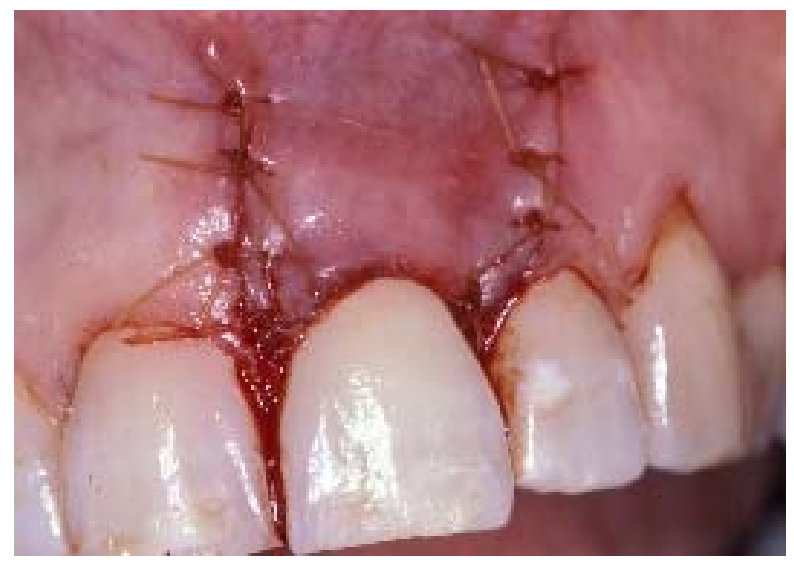

b

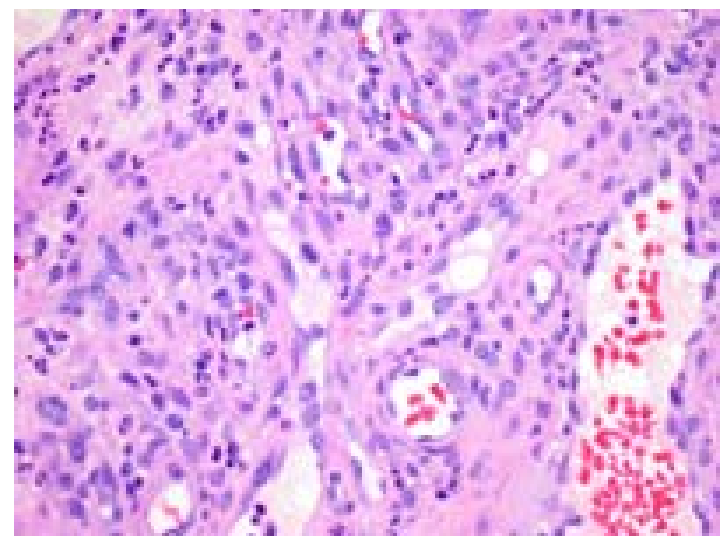

d

Fig. (2). Pyogenic granuloma. (a) Initial presentation of a pyogenic granuloma at the maxillary anterior teeth, facial view (note recession present at tooth \#9). (b) Surgical excision of lesion and coronal positioning of gingival margin to correct recession defect. (c) 21 days postsurgery with gingival recession defect corrected and normal marginal tissue. (d) Pyogenic granuloma with underlying vascularity (20 X power). 
Local irritation is usually present, such as calculus, caries, defective restorations, or trauma (Fig. 3). This 37 year old male presented with a red lesion at the papilla between teeth \#8 and 9 of unknown duration. The patient also complained of an increasing separation of the contact between the teeth. A surgical excision was performed to remove the entire papilla and underlying attachment to the crestal bone. Root planing was done to remove subgingival calculus and a mucoperiosteal flap was elevated and coronally positioned to recreate an interproximal papilla of normal tissue. The healing at 21 days shows a closure of the contact between teeth $\# 8$ and 9 and healthy tissue. No recurrence has been seen over the past five years.

Histologically, the PF consists of hyperplastic fibrous tissue with a varying degree of vascularity. The nonulcerated lesion is covered by a layer of keratinized squamous cell epithelium. The tissue mass consists of bundles of collagen fibers often arranged in radiating, circular or haphazard fashion. The vast majority is exceedingly dense fibrous connective tissue with poor vascularity and few chronic inflammatory cells present (Fig. 3). In the presence of trauma, the PF may become ulcerated and infiltrated with inflammatory cells.

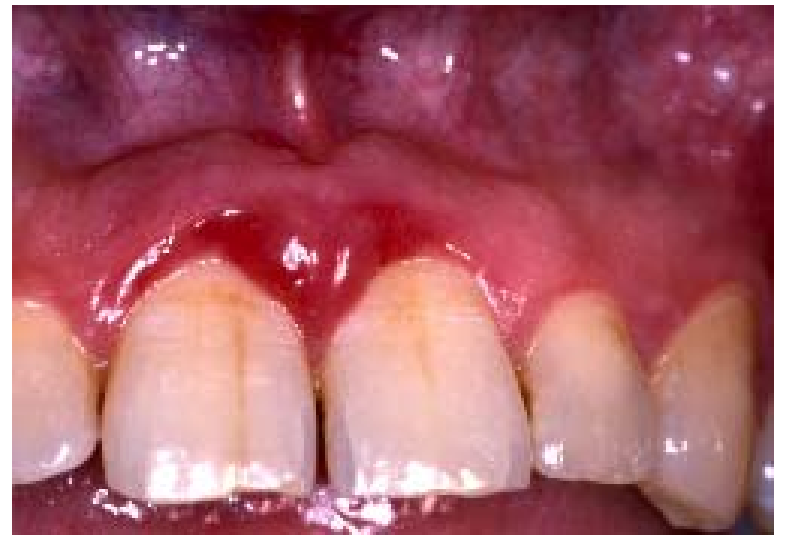

$\mathrm{a}$

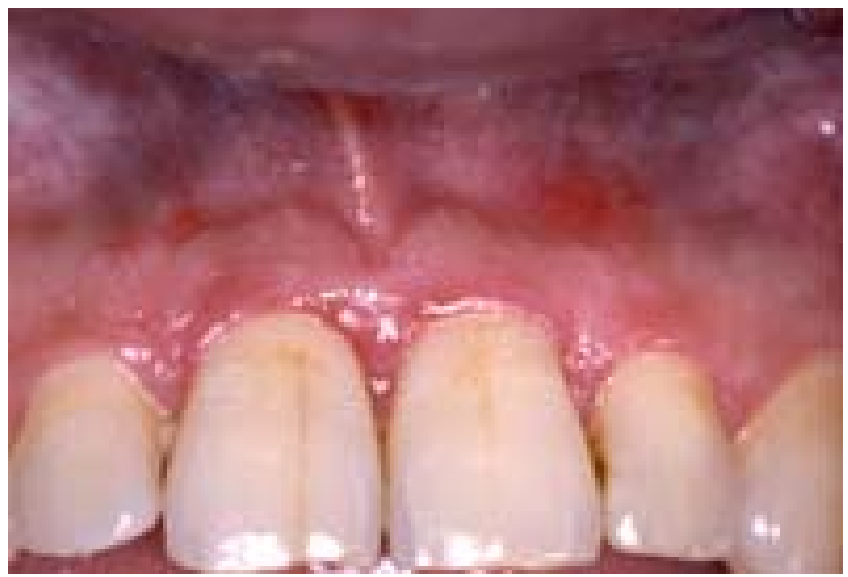

C
The treatment of choice is conservative excision of the lesion to include the gingival connective tissue base and removal of the irritating factors. Often a defective restoration or an open contact may be involved; to reduce recurrence of the lesion this should also be addressed. Since trauma is a common etiologic factor, any parafunctional occlusal habits should be investigated and treated in addition to removal of the PF. The carbon dioxide laser can effectively excise the lesion and has been shown to allow diagnostic microscopic evaluation with minimal distortion of the biopsy sample [9]. The advantages of laser excision are minimal post-surgical pain and no need for suturing the biopsy site. The recurrence rate is low once the irritating factors are eliminated.

\section{PERIPHERAL GIANT CELL GRANULOMA}

The peripheral giant cell granuloma (PGCG) is the least frequently seen of the common reactive lesions of the gingiva, reported in only $5 \%$ of the biopsy reports according to the published literature [4,5]. It originates from the periosteum or periodontal ligament as a result of local irritation or chronic trauma. The PGCG is a deep red to reddish blue raised lesion which is sessile or pedunculated, seen more frequently in the mandible (Fig. 4). The lesion can develop at any age, but is most commonly reported in the

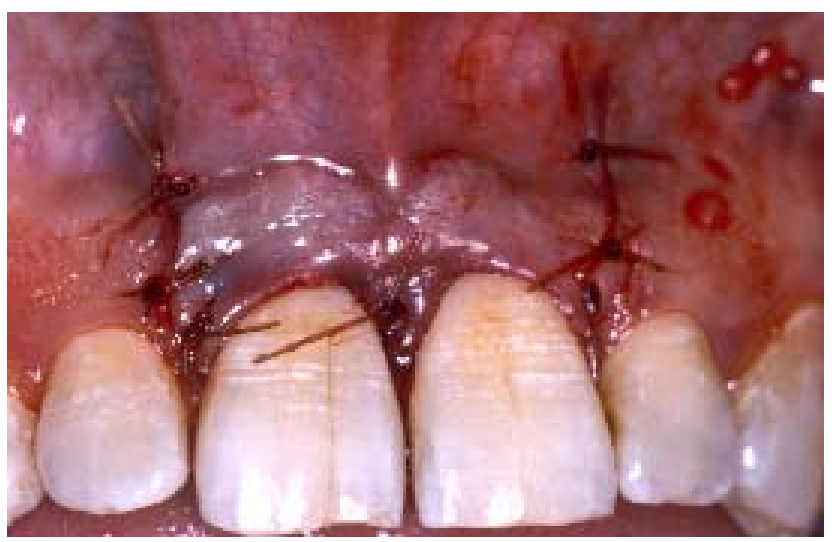

b

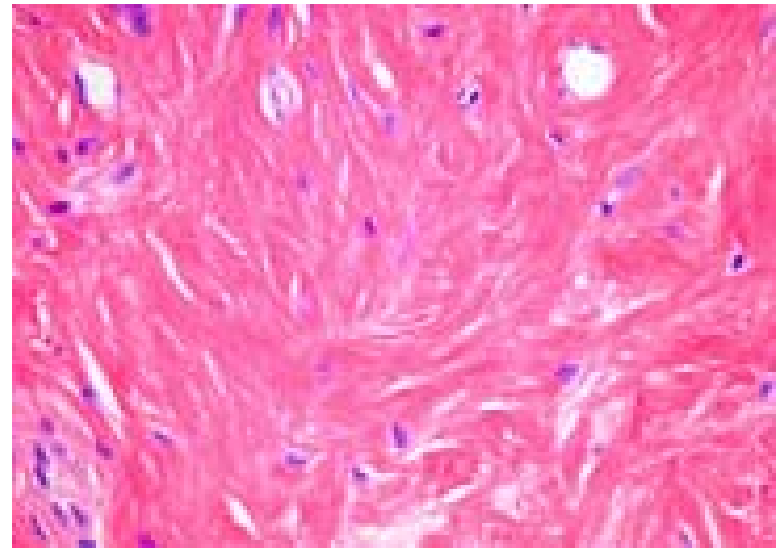

d

Fig. (3). Fibroma. (a) Initial presentation of a peripheral fibroma at the maxillary anterior teeth, facial view. (b) Surgical excision of lesion with coronal positioning of normal gingiva. (c) 21 days post-surgery with normal gingival margins. (d) Peripheral fibroma showing underlying dense collagen bundles in a haphazard fashion (20 X power). 
fourth to sixth decade of life. PGCG is a soft tissue lesion that rarely involves the underlying bone. However, superficial erosion or cupping of the underlying bone has been reported. In addition a widening of the periodontal ligament space is often seen with mobility of the adjacent teeth.

The microscopic features of PGCG include a nonencapsulated tissue mass composed of a reticular and fibrillar connective tissue containing abundant plump ovoid or spindle shaped mesencymal cells. The PGCG is covered by keratinized squamous cell epithelium which is often ulcerated. The most obvious feature is a large number of multinucleated giant cells scattered throughout the lesion. The histogenesis of the giant cells is unknown, they appear to be non-functional as phagocytes or bone resorbers [6]. The lesions contain abundant small capillaries and hemorrhagic foci with hemosiderin deposition. A diffuse inflammatory infiltrate is often seen containing both acute and chronic cells. Many of the lesions contain islands of dystrophic calcification consisting of both newly formed bone and mature calcified material.

Treatment of PGCG involves surgical resection down to underlying bone with elimination of the etiologic factors. The surgical excision should include debridement of the boney walls and curettage of the periosteum. In addition, scaling and curettage of the adjacent teeth and periodontal ligament is involved. Although the carbon dioxide laser has been reported to effectively remove the PGCG it must also be combined with exposure of the underlying bone through reflection of a mucoperiosteal flap [14]. This limits the usefulness of the laser for lesions with adjacent bone involvement since the carbon dioxide laser is contraindicated for hard tissue $[15,16]$. The recurrence rate is low and generally reported to be less than $10 \%$, when the irritating factors are eliminated. This 45 year old male presented with a history of diabetes mellitus type 2 controlled with

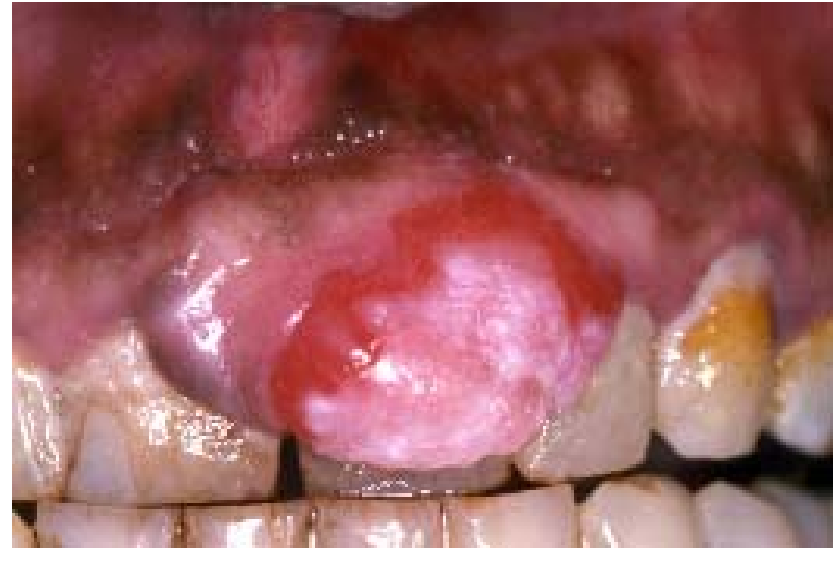

a

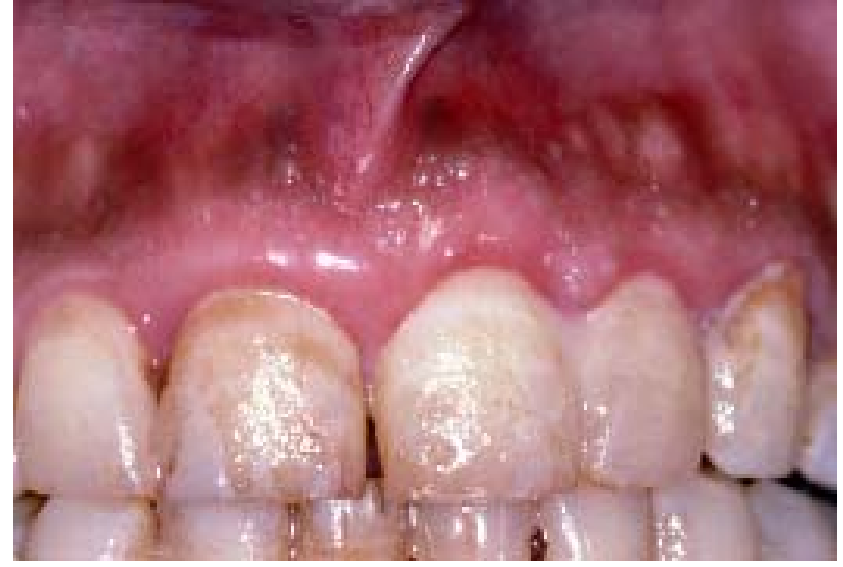

b

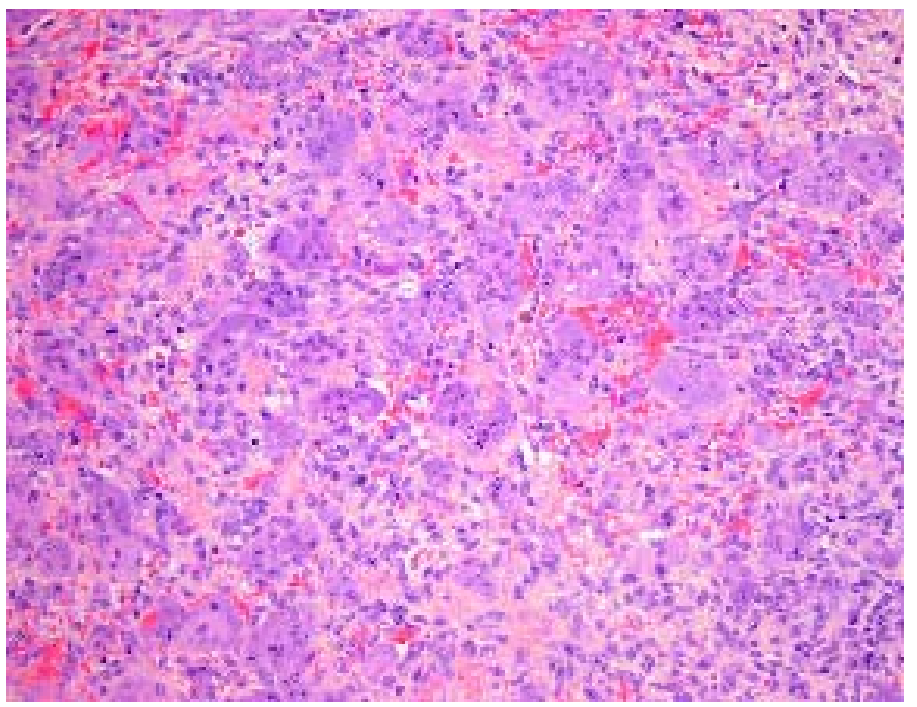

C

Fig. (4). Peripheral giant cell granuloma. (a) Original presentation of a peripheral giant cell granuloma at the maxillary anterior teeth, facial view. (b) 21 days post-surgery. The PGCG was surgically excised with scalpel and $\mathrm{CO}_{2}$ laser. (c) Central portion of a PGCG showing multinucleated giant cells and extravasated red blood cells (10 X power). 
medications. He stated the lesion had been present for several years and recently increased in size. The patient had not been under regular dental care for many years. The lesion was removed intact with a scalpel to include the underlying periodontal attachment and the teeth were root planed to remove calculus. The carbon dioxide laser was used to contour the gingival tissues and obtain hemostasis. Despite plaque accumulation the surgical site is healing well after 21 days. Recurrence will be dependent upon improved plaque control and regular dental care (Fig. 4). The importance of obtaining a histologic diagnosis is exemplified with this case, since dysplastic changes may occur with any long standing oral lesion.

\section{PERIPHERAL OSSIFYING FIBROMA}

The peripheral ossifying fibroma (POF) is a relatively common reactive lesion of the gingiva, accounting for $9.6 \%$ of all biopsy reports of gingival lesions [17]. The nomenclature for this lesion however has been confusing and is often reported as a peripheral fibroma with calcification or a peripheral odontogenic fibroma. The issue has become more clouded since the World Health Organization classification system recognized a peripheral odontogenic fibroma as a distinct and different entity from the POF. Current literature refers to this lesion as the peripheral ossifying fibroma (WHO type) and it is recognized separately from the POF of gingiva [18-20]. The POF is thought to originate from the superficial periodontal ligament and is found most often in the anterior maxilla. There is a predilection for females in the second to fourth decade of life. This raised lesion may appear smooth and pink or ulcerated and erythematous. The clinical appearance may be identical to the peripheral fibroma and both are associated with local irritating factors. Due to the higher recurrence rate of the POF it is essential to obtain a histologic diagnosis for this lesion, as is recommended for all reactive gingival lesions.

Histologically, the POF is more cellular than the peripheral fibroma and less vascular than the pyogenic granuloma. Fibrous proliferation with large numbers of fibroblasts are seen associated with formation of mineralized product that may include bone, cementum-like material, dystrophic calcification or a combination of each. The surface of the lesion may be intact or ulcerated stratified squamous epithelium. The characteristic feature of the POF is highly cellular connective tissue containing foci of calcified material. The origin of the POF from the periodontal ligament has been suggested due to its exclusive occurrence in the interdental papilla, its proximity to the periodontal ligament, and the presence of oxytalan fibers within the mineralized matrix of the lesion [18, 21].

Surgical excision of the POF is required with aggressive curettage of the underlying periosteum to include scaling of the adjacent teeth and periodontal ligament space to reduce recurrence [22]. The rate of recurrence is high, around $16 \%$, presumably due to superficial excision and incomplete removal of the local irritants [23-25]. There is a tendency with the $\mathrm{CO}_{2}$ laser to do incomplete removal of the POF since the ablation is in close proximity to the root surfaces. Also tissue destruction with the carbon dioxide laser is a shallow wound in tissue with high water content. Therefore, laser ablation of the interproximal soft tissue down to periosteum requires multiple passes which can damage adjacent root surfaces and may have limited access for the laser handpiece. This 21 year old female presented with a raised lesion on the palate between teeth \#10 and 11 of unknown duration (Fig. 5). Due to excellent access to the lesion from a diastema present interproximally, the carbon dioxide laser was used to excise the lesion at the stalk. Following ablation by the laser into the periodontal ligament space a carbonized layer was left to serve as a biologic bandage. The 28 day healed result shows no evidence of remaining lesion and no periodontal attachment loss in the site. No recurrence has been reported in this case over the past ten years.

Reflection of a mucoperiosteal flap is often required to treat the cosmetic defect resulting from complete excision of the lesion (Fig. 6). This allows for correction at the time of excision through coronal advancement of the non-involved healthy gingiva and often is combined with a connective tissue graft for a better esthetic result [23]. Long term follow-up is recommended due to the higher recurrence rate of the POF compared with other reactive lesions.

\section{LOCALIZED JUVENILE SPONGIOTIC GINGIVAL HYPERPLASIA}

This lesion is considered a unique and distinctive form of inflammatory gingival hyperplasia seen in young patients (average age 11.8 years), predominantly female and generally found in the maxillary anterior region This type of lesion was first described by Darling et al. as juvenile spongiotic gingivitis [2]. After the investigation of a larger sample size by Chang et al. the more accurate term LJSGH had been suggested [3]. In her retrospective study LJSGH had been identified in biopsy reports covering 20 years, but had not been classified as a distinct entity until recently. It appears as a bright red raised overgrowth with a papillary or finely granular surface, however, it does not seem to be a plaque related lesion. The lesion presents as a small (average size was $6 \mathrm{~mm}$ ), localized and easily bleeding overgrowth on the gingiva of a child. It is usually given the clinical diagnosis of pyogenic granuloma and frequently seen in conjunction with orthodontic brackets, which may be purely coincident with the patient population. This 15 year old male presented in full orthodontic brackets with a red lesion of several weeks duration. The lesion was not painful, but bled easily. Surgical excision was performed by scalpel for histologic analysis with subsequent gingivectomy by the carbon dioxide laser to the adjacent area to remove hyperplastic gingiva and obtain hemostasis (Fig. 7). The area has healed with resolution of the lesion and no recurrence over the past year.

The histologic presentation is an exophytic lesion with a subtle papillary architecture composed of interconnecting bands of epithelial hyperplasia. The histology is unique and characterized by prominent intercellular edema (spongiosis) and neutrophilic exocytosis. The presence of highly vascular connective tissue cores is seen containing mostly acute, but with some chronic inflammatory cells (Fig. 8). LJSGH displays as a gingival overgrowth rather than a pure inflammatory process with minimal to no tissue swelling. Therefore, the terminology is more appropriately an 


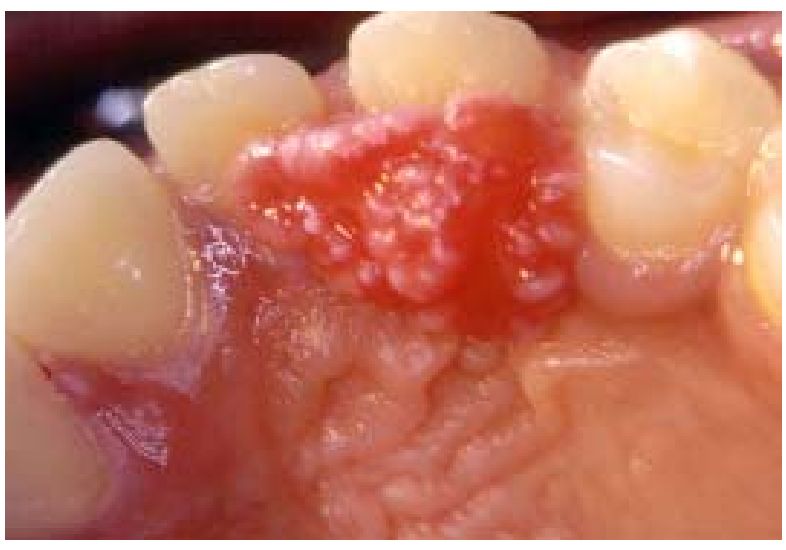

a

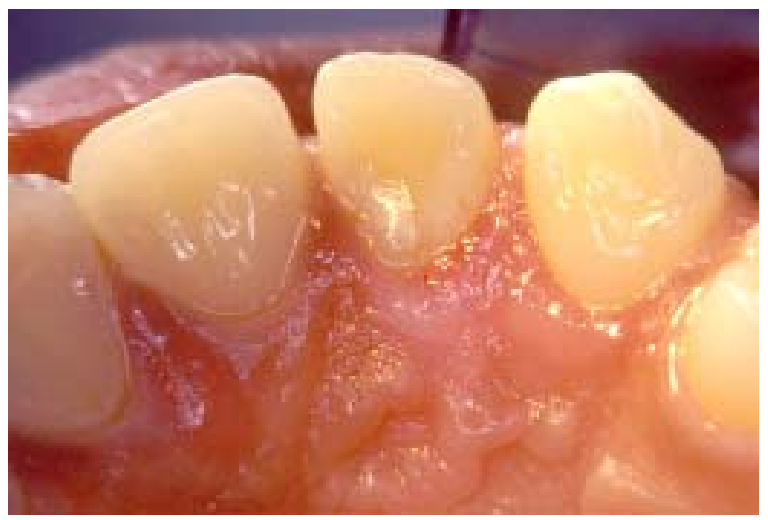

C

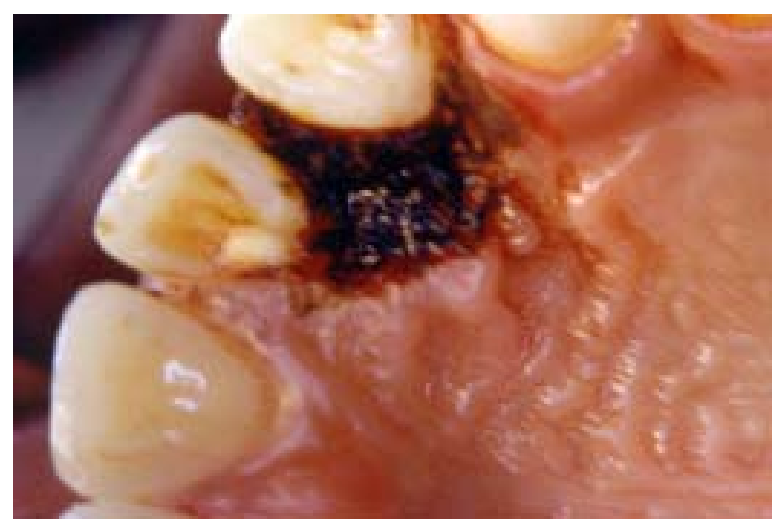

$\mathrm{b}$

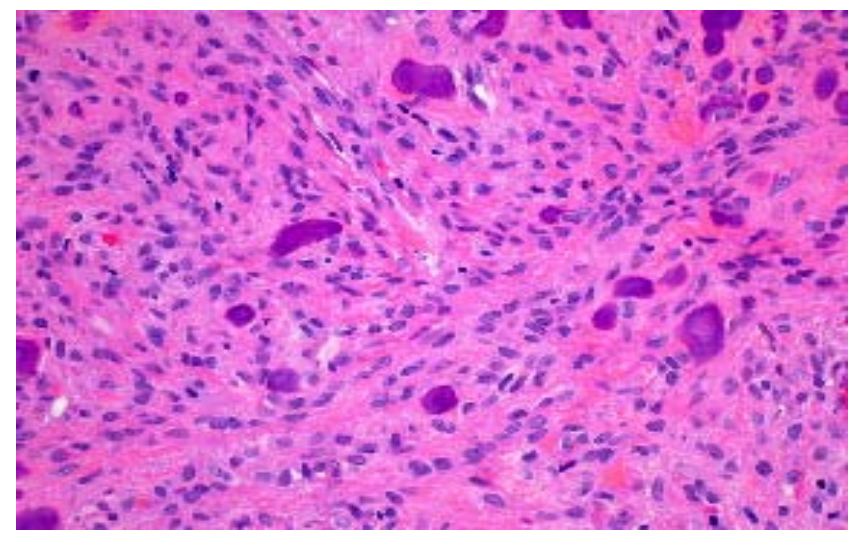

d

Fig. (5). Peripheral ossifying fibroma. (a) Original presentation of a peripheral ossifying fibroma at the maxillary anterior teeth, palatal view. (b) Surgical excision by $\mathrm{CO}_{2}$ laser to include ablation into the periodontal ligament space. (c) 28 days post-surgery showing no recurrence of the POF lesion and no attachment loss. (d) POF showing cellular stroma with cementoid calcifications (20 X power).

epithelial hyperplasia than gingivitis. The etiology is unknown and the lesion does not respond to periodontal treatment showing a lack of association with plaque or calculus. Darling et al. compared juvenile spongiotic gingivitis/LJSGH with puberty gingivitis and found several distinguishing features including a lack of immunoreactivity for estrogen and progesterone receptors in LJSGH [2].

Treatment for LJSGH is conservative surgical excision and the carbon dioxide laser is ideal for treating this lesion. Since the raised mass is highly vascular, laser ablation and its benefit of hemostasis during surgical removal is imperative to achieve complete elimination of the hyperplastic tissue. The young age of the patient makes laser ablation a preferred and very efficient procedure, well tolerated by this population of patients. Recurrence is rare and when it does occur, may be due to incomplete removal of the lesion.

\section{DISCUSSION}

The use of lasers in removal of soft tissue lesions has been an accepted practice for many years. The carbon dioxide laser has been cited in the surgical literature for dental use since the 1970's and is the most commonly used laser in dentistry for soft tissue ablation or excision. The benefits include the following: 1) high visibility during surgery for more accurate removal of pathology; 2) minimal contact with the pathologic tissues; 3 ) the laser sterilizes as it cuts to reduce transmission of disease; and 4) it seals the minor vasculature and lymphatics during ablation. Laser surgery results in less post-operative swelling, reduced pain, less damage to adjacent soft tissues, less wound contraction or scarring due to minimal connective tissue response, reportedly reduced risk of post-operative infection, and possibly less recurrence of the lesion $[9,16]$. Bornstein et al. also report of 139 patients treated by carbon dioxide laser excision of soft tissue lesions that there was no interference in diagnostic ability from thermal damage to the biopsy specimens. These benefits make the use of lasers for treatment of reactive lesions of the gingiva a potent addition to the surgical armamentarium.

The carbon dioxide laser has a great affinity for water and the laser energy is highly absorbed in oral soft tissues. This leaves a shallow wound at the target site with minimal thermal damage to underlying tissue and sharp wound margins. This precise tissue destruction can also result in partial or incomplete removal of the base of the pathologic lesion which can lead to recurrence [26]. It is incumbent on the therapist to obtain complete removal of gingival lesions 


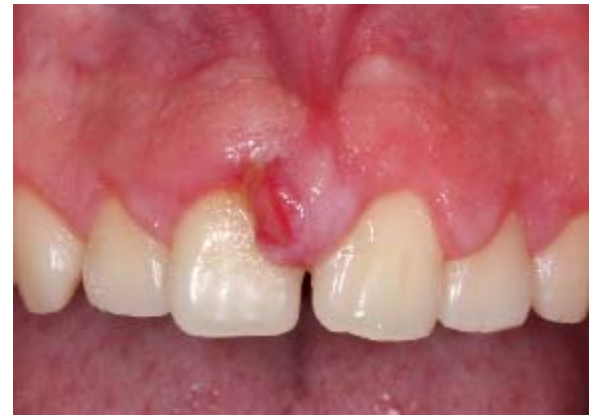

a

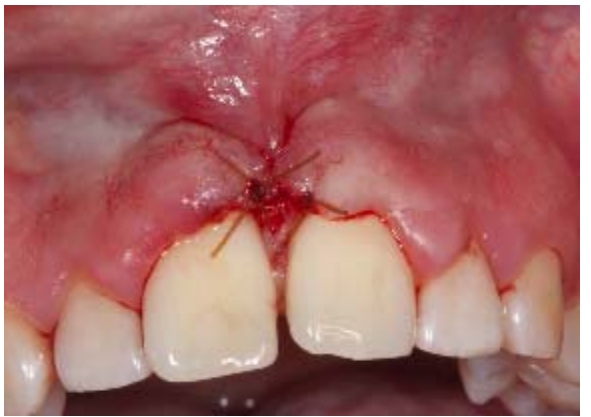

b

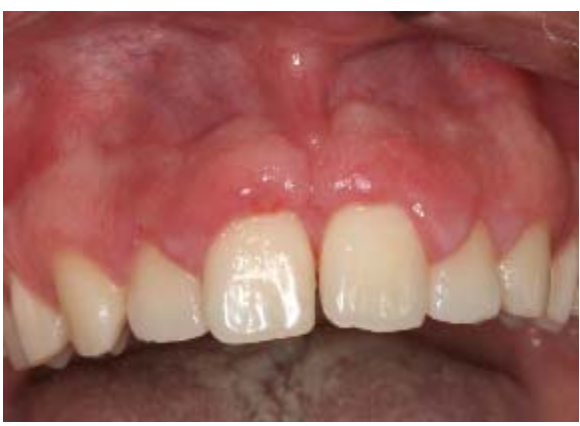

$\mathrm{C}$

Fig. (6). Peripheral ossifying fibroma. (a) Original presentation of POF at the maxillary anterior teeth, facial view. (b) Surgical excision by scalpel with debridement of periodontal ligament. (c) 21 days post-surgery (Photos courtesy of Dr. Stephen Walker).

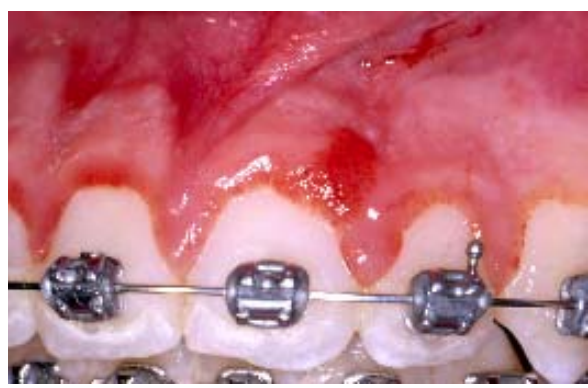

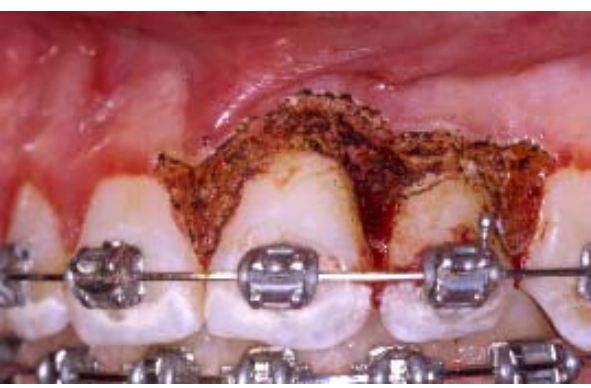

b

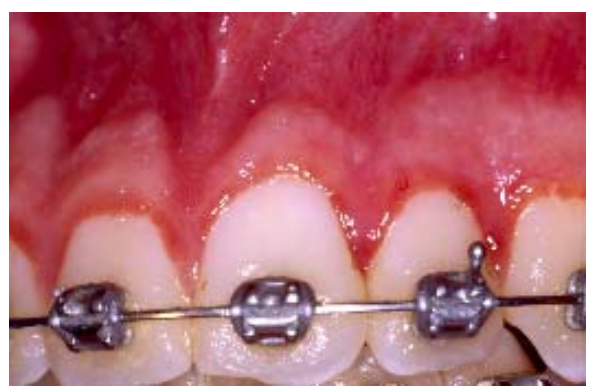

C

Fig. (7). Localized juvenile spongiotic gingival hyperplasia. (a) Original presentation of localized juvenile spongiotic gingival hyperplasia at the maxillary arch. (b) Surgical ablation by $\mathrm{CO}_{2}$ laser of LJSGH lesion and gingivoplasty to hyperplastic papilla. (c) 14 days post-surgery (note mild gingivitis present).

down to normal underlying tissue, which may make the scalpel a preferred instrument for some lesions. Additionally, the lesions arising from the periodontal ligament require thorough debridement of the stem cells in the ligament space and periosteum to reduce the recurrence rate. Surgical excision will often involve reflection of a full thickness mucoperiosteal flap to access this portion of the lesion. This is especially true for the peripheral ossifying fibroma as discussed by Walters et al. [23] and Buduneli et al. [22]. The reflection of a full thickness flap will also provide the surgeon with an opportunity to repair any soft tissue mucogingival defect at the time of excisional biopsy, as seen in Figs. $(\mathbf{2}, \mathbf{3})$. 


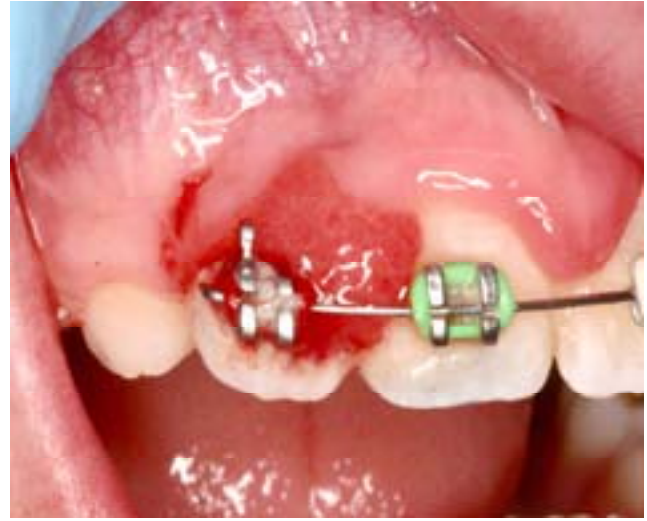

a

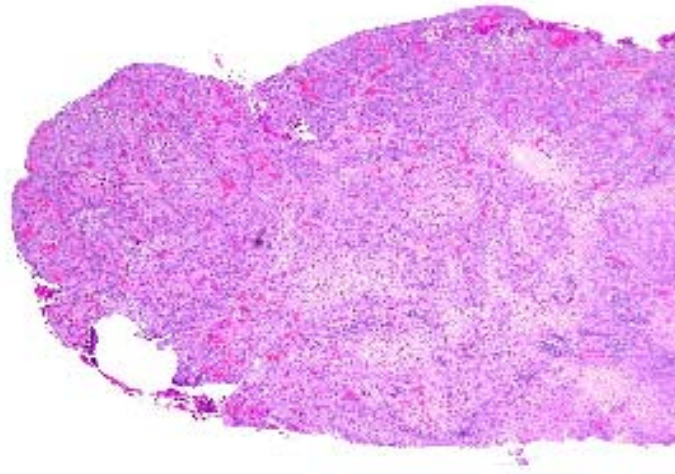

b

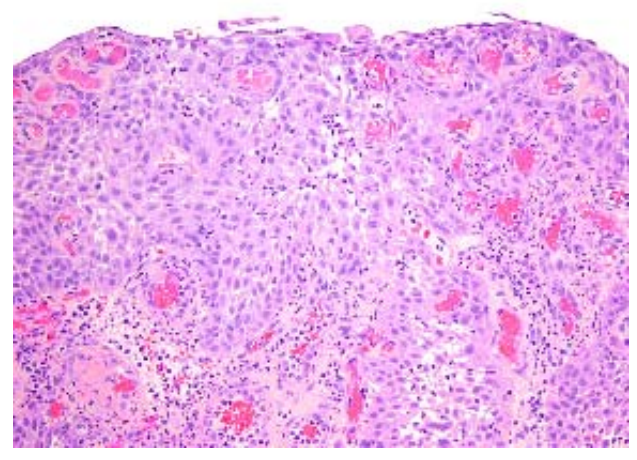

C

Fig. (8). Localized juvenile spongiotic gingival hyperplasia. (a) Original presentation of LJSGH as a localized lesion at the maxillary arch, facial view. (photo courtesy of Dr. Harvey Kessler). (b) LJSGH lesion showing cobblestone surface and proliferation of epithelium (2 X power). (c) LJSGH lesion showing proliferation of sulcular epithelium with exocytosis and dilated blood vessels within connective tissue cores $(10 \mathrm{X}$ power).

The gingival lesions described in this paper often have similar clinical appearances and have a 2:1 predilection for females. They also have similar patient complaints of enlargement, bleeding upon provocation, and occasionally gingival pain. Some authors believe the differing histologic pictures are a spectrum of a single lesion in different stages of maturation $[7,20]$. Despite their similarities all reactive gingival lesions show differences in age, type, location, duration, and histologic features. Imperative in the treatment of reactive gingival lesions is the complete removal of local irritants such as defective restorations, calculus, or trauma. Successful treatment involves obtaining an accurate diagnosis through histopathologic analysis, complete removal of the gingival lesion, and addressing the local irritants with follow-up care, as well as dental hygiene maintenance to prevent or treat recurrence.

\section{ACKNOWLEDGEMENTS}

The author reports no conflict of interest related to this study or the recommendation of alternative surgical treatment methods. The author wishes to thank Dr. Harvey Kessler for his assistance in preparation of this manuscript.

\section{REFERENCES}

[1] Neville BW, Damm DD, Allen CM, Bouquot JE. Oral and maxillofacial pathology. 3rd ed. St. Louis: Saunders 2009.
[2] Darling MR, Daley TD, Wilson A, Wysocki GP. Juvenile spongiotic gingivitis. J Periodontol 2007; 78(7): 1235-40.

[3] Chang JY, Kessler HP, Wright JM. Localized juvenile spongiotic gingival hyperplasia. Oral Surg Oral Med Oral Pathol Oral Radiol Endod 2008; 106(9): 411-8.

[4] Zhang W, Chen Y, An Z, Geng N, Bao D. Reactive gingival lesions: A retrospective study of 2,439 cases. Quintessence Int 2007; 38(2): 103-10

[5] Kfir Y, Buchner A, Hansen LS. Reactive lesions of the gingiva: a clinicopathological study of 741 cases. J Periodontol 1980; 51(11): 655-61.

[6] Binnie WH. Periodontal cysts and epulides. Periodontology 2000 1999; 21: 16-32.

[7] Prasad S, Reddy SB, Patil SR, Kalburgi NB, Puranik RS Peripheral ossifying fibroma and pyogenic granuloma. N Y State Dent J 2008; 74(2): 50-2.

[8] Daley TD, Nartey NO, Wysocki GP. Pregnancy tumor: an analysis. Oral Surg Oral Med Oral Pathol 1991; 72(2): 196-9.

[9] Bornstein MM, Winzap-Kälin C, Cochran DL, Buser D. The $\mathrm{CO}_{2}$ laser for excisional biopsies of oral lesions: a case series study. Int J Periodontics Restorative Dent 2005; 25(3): 221-9.

[10] Frame JW. Removal of oral soft tissue pathology with the $\mathrm{CO}_{2}$ laser. J Oral Maxillofac Surg 1985; 43: 850-5.

[11] White JM, Chaudry SI, Kudler JJ, Sekandari N, Schoelch ML, Silverman S. Nd:YAG and $\mathrm{CO}_{2}$ laser therapy of oral mucosal lesions. J Clin Laser Med Surg 1998; 16(6): 299-304.

[12] Salum FG, Yurgel LS, Cherubini K, De Figueiredo MAZ, Medeiros IC, Nicola FS. Pyogenic granuloma, peripheral giant cell granuloma and peripheral ossifying fibroma: retrospective analysis of 138 cases. Minerva Stomatol 2008; 57(3): 227-32.

[13] Esmeili T, Lozada-Nur F, Epstein J. Common benign oral soft tissue masses. Dent Clin N Am 2005; 49: 223-40. 
[14] Chaparro-Avendaño AV, Berini-Aytés L, Gay-Escoda C. Peripheral giant cell granuloma: a report of five cases and review of the literature. Med Oral Patol Oral Cir Bucal 2005; 10: 48-57.

[15] Pick RM, Colvard MD. Current status of lasers in soft tissue dental surgery. J Periodontol 1993; 64(7): 589-602.

[16] Rossmann JA, Cobb CM. Lasers in periodontal therapy. Periodontology 2000 1995; 9: 150-64.

[17] Layfield LL, Shopper TP, Weir JC. A diagnostic survey of biopsied gingival lesions. J Dent Hyg 1995; 69: 175-9.

[18] Gardner DG, The peripheral odontogenic fibroma: an attempt at clarification. Oral Surg Oral Med Oral Pathol 1982; 54(1): 40-8.

[19] Wright JM. Oral and maxillofacial pathology case of the month. Texas Dent J 2000; 117(11): 62-9.

[20] Buchner A, Hansen LS. The histomorphologic spectrum of peripheral ossifying fibroma. Oral Surg Oral Med Oral Pathol 1987; 63(4): 452-61.

[21] Mesquita RA, Sousa SC, de Araújo NS. Proliferative activity in peripheral ossifying fibroma and ossifying fibroma. J Oral Pathol Med 1998; 27(2): 64-7.
Buduneli E, Buduneli N, Ünal T. Long-term follow-up of peripheral ossifying fibroma: Report of three cases. Periodontal Clin Invesigt 2001; 23(1): 11-4

[23] Walters JD, Will JK, Hatfield RD, Cacchillo DA, Raabe DA. Excision and repair of the peripheral ossifying fibroma: a report of 3 cases. J Periodontol 2001; 72(7): 939-44.

[24] Farquhar T, MacLellan J, Dyment H, Anderson RD. Peripheral ossifying fibroma: a case report. J Can Dent Assoc 2008; 74(9): 809-12.

[25] Carrera GI, Berini-Aytés L, Gay-Escoda C. Peripheral ossifying fibroma: Report of a case and review of the literature. Med Oral 2001; 6(2): 135-41.

[26] Tamarit-Borràs $\mathrm{M}$, Delgado-Molina E, Berini-Aytés L, GayEscoda, C. Removal of hyperplastic lesions of the oral cavity: a retrospective study of 128 cases. Med Oral Patol Oral Cir Bucal 2005; 10: 151-62.

(C) Jeffrey A. Rossmann; Licensee Bentham Open.

This is an open access article licensed under the terms of the Creative Commons Attribution Non-Commercial License (http: //creativecommons.org/licenses/by-nc/ 3.0/) which permits unrestricted, non-commercial use, distribution and reproduction in any medium, provided the work is properly cited. 